\title{
Fault Location in Multi-Terminal HVDC Networks Based on Electromagnetic Time Reversal with Limited Time Reversal Window
}

\author{
Reza Razzaghi, Mario Paolone, Farhad Rachidi \\ École Polytechnique Fédérale de Lausanne EPFL \\ Lausanne, Switzerland \\ \{reza.razzaghi, mario.paolone, \\ farhad.rachidi\}@epfl.ch
}

\author{
Justine Descloux \\ RTE-CNER-DCCL \\ Paris, France \\ justine.descloux \\ @rrte-france.com
}

\author{
Bertrand Raison, Nicolas Retière \\ Univ. Grenoble Alpes, G2ELab \\ Grenoble, France \\ \{bertrand.raison, \\ nicolas.retiere\}@g2elab.grenoble-inp.fr
}

\begin{abstract}
This paper aims at proving the application of the fault location method based on the Electromagnetic Time Reversal (EMTR) to multi-terminal HVDC (MTDC) networks. In particular, the paper integrates the EMTR fault location technique with the protection scheme recently proposed within the EU project TWENTIES. Further, in view of the peculiarity of the fast protection schemes required by HVDC applications, the paper discusses the performances of the EMTR-based fault location technique by using limited time-windows over which the fault-generated electromagnetic transients are time-reversed. The paper also discusses the advantage of the EMTR fault location related to the use of a single observation point to the case of MTDC grids. Indeed, such a peculiarity may represent a major advantage avoiding necessary time synchronization between the MTDC-fault recording stations and might represents, also, a backup protection system. The performances of the proposed method are validated by numerical simulations obtained using the EMTP-RV simulation environment where electromagnetic fault-transients are reproduced with reference to the MTDC benchmark network of the project TWENTIES.
\end{abstract}

Keywords- Fault location; electromagnetic time-reversal; multi-terminal HVDC networks; electromagnetic fault transients; traveling waves.

\section{INTRODUCTION}

In modern power systems, HVDC transmission lines are widely used as an alternative for conventional ones. As known, these transmission systems enable the possibility of transferring high amount of power over long distances as well as interconnecting large parks of renewable-type generators typically composed of off-shore wind farms. Due to the nature of the HVDC systems, the protection and fault location in HVDC transmission systems are crucial issues [1-3] and the major challenge is represented by the need of identifying the faulted line within time windows of few milliseconds.

For the case of AC transmission lines, as summarized in [4], existing fault location methods can be classified in two general categories: (i) analysis of pre- and post-fault voltage/current phasors and, (ii) analysis of fault-originated electromagnetic transients of currents and/or voltages.
For the case of HVDC transmission, the first category of fault location methods cannot be obviously applied. Thus, in general, fault location methods for HVDC transmission are based on traveling waves [1]. In this respect, existing traveling wave-based fault location methods for two-terminal HVDC transmission systems are classified in two further general categories (e.g., [5,6]): (i) methods based on one terminal measurement, and (ii) methods based on two terminal measurements. Compared to the methods based on oneterminal measurement, two-terminal measurement methods provide, as expected, higher fault location accuracy. However, they require synchronization between the measurements at each terminal, adding a non-negligible complexity to the system [6].

Multi-terminal DC networks (MTDC) provide more reliability of the interconnected network. However, the protection and fault location are even more challenging for these types of networks compared to point-to-point HVDC links. Generally, they require fast communication links [7]. In particular, due to the multiple paths for the travelling waves in the MTDC network, the fault location problem is more complicated compared to the case of two-terminal HVDC lines.

In order to provide a solution to this problem, this paper presents a procedure based on the theory of Time-Reversal Process [8-15] applied to traveling waves in transmission lines $[16,17]$ using measurements at one terminal only.

The basic idea of the EMTR is to take advantage of the reversibility in time of the wave equation. The applicability of the EMTR technique to locate faults in transmission lines was recently presented in $[16,17]$. It was shown that, when electromagnetic transients observed in specific observation points are time-reversed and back-injected into the system, or in a simulated version of it, they would converge to the source (fault) location.

The purpose of this paper is to extend a modified version of the algorithm presented in [16] as a fault locator tool for MTDC networks. It uses a single-measurement terminal. Further, we will show that, even with limited time-reversed windows adopted to observe the post-fault transients, the 
proposed fault location method is still robust with limited fault location errors.

The proposed fault location technique is composed of two steps. First, the faulted DC line is identified using the approach proposed in [18]. Then, this information is used in a second step to select a specific observation point at which, the recorded electromagnetic transients, are time-reversed and back-injected into an emulated network model for different guessed fault locations. In particular, due to the fast protection system of MTDC networks, which yields in opening the faulted line breakers in only a few milliseconds, the proposed method is based on the use of a limited time reversal window, (normally the first $5 \mathrm{~ms}$ of the recorded transient signals are used in the time reversal process). For each guessed fault location, the energy of the current signal flowing through it is evaluated in order to infer the potential fault location.

The structure of the paper is as follows. Section II presents briefly the protection scheme assumed to be used for detecting the faulted line in the MTDC network. Then, Section III describes the basic concept of the EMTR theory. In Section IV, the EMTR-based fault location algorithm is presented for the case of MTDC networks where a limited time reversal window is used. Section V illustrates the application of the proposed method using EMTP-simulated cases referring to a MTDC network. Section VI discusses the sensitivity of the fault location accuracy as a function of the length of the timereversal window of the measured electromagnetic transients and, finally, Section VII concludes the paper with a summary and conclusions.

\section{PROTECTION SCHEME FOR IDENTIFYING THE FAULTED LINE INTO A MTDC NETWORKS}

The multi-terminal DC grid under study has been defined during the European TWENTIES project and consists of five nodes connected to $\mathrm{AC}$ sources or $\mathrm{AC}$ grids, and five bipolar undersea power cables $( \pm 320 \mathrm{kV})$ as depicted in Fig.1. However, in this paper we have assumed that these links are composed by overhead $(\mathrm{OH})$ bipolar transmission lines ${ }^{1}$. As known, two types of faults can occur: either pole-to-ground faults, or pole-to-pole faults.

The objective of the protection scheme is to protect the components of the grid but, also, to be selective, which means that, if a fault occurs on a cable/OH line, only the faulted line has to be isolated from the healthy part of the grid. To ensure this selectivity, a protection relay needs to be installed at each end of line, on each pole, and on both sides of the busbars, as shown in Fig.1. Each protection relay is associated with a DC circuit breaker and a current sensor.

Note that DC line insulation faults only are considered here. The major constraint on the protection strategy is the very short response time required for the algorithms, which are relative to the components resistance under fault conditions. Indeed, the anti-parallel diodes of the VSCs can only withstand a current up to $2 \mathrm{pu}$. Thus, the protections have a very short time to operate (in the range of $10 \mathrm{~ms}$ ) including the time to detect the

\footnotetext{
${ }^{1}$ Note that, the system is symmetrical bipolar without metallic return.
}

fault, to discriminate between the faulted and healthy parts of the grid and the opening time of the circuit breakers.

The proposed protection plan consists of three different protections:

- a main cable protection,

- a busbar protection,

- a backup protection.

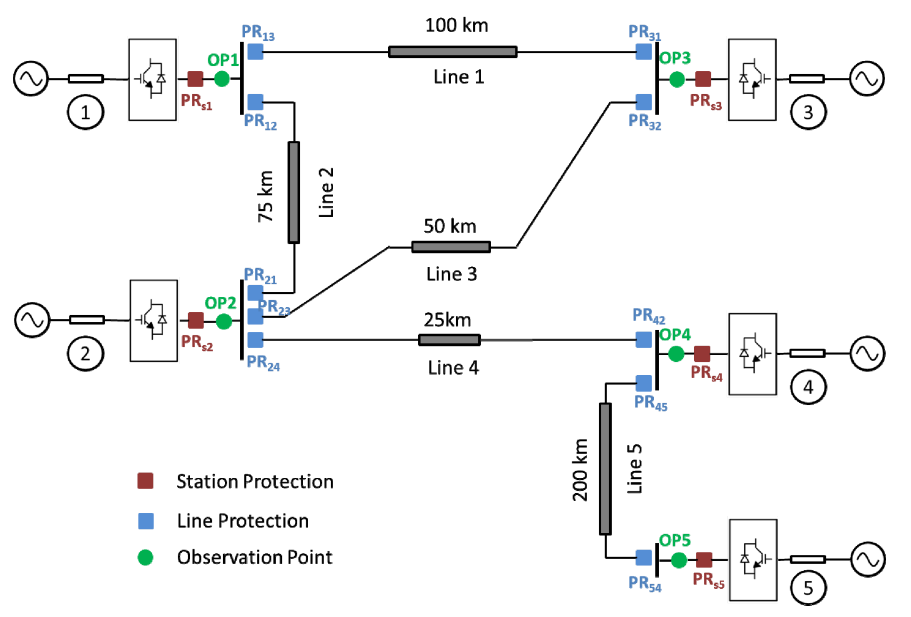

Fig. 1. Schematic representation of the MTDC network.

The main protection algorithm has been proved to be selective in [18]. It makes it possible to detect a fault occurring on a cable and to send a tripping order to DC circuit breakers of each end of the faulted link. The busbar protection is set to protect the grid against DC faults occurring between each sensor, and sends a tripping order to both feeders and source breakers. Then, a backup algorithm trips all breakers of the busbar in case of failure of the main protection. The main cable protection algorithm is detailed below.

The main protection consists of the selective detection of faults occurring on a DC cable. The selectivity of the algorithm is achieved by using a telecommunication link (optic fiber) set up in parallel to each power cable. Hence, each line protection exchanges its own information with the opposite line-end protection. Considering that fact, several algorithms can be implemented. The easiest one, in terms of computation time and robustness, has been chosen. It is based on the evaluation of the differential current, and is adapted to the dynamics of the signals under fault conditions in DC grids. The algorithm governing the protection is displayed below (see [18] for further details):

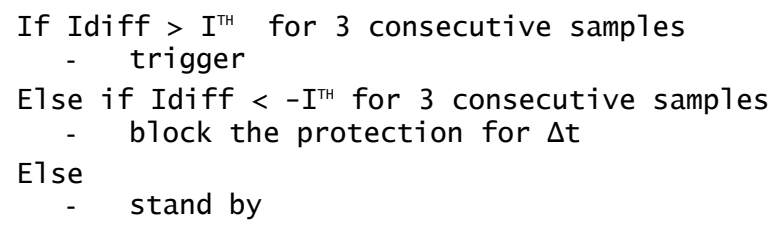

where $\mathrm{I}^{\mathrm{TH}}$ is a threshold and Idiff is the differential current defined as the difference between the currents measured at the 
ends of the cable. An ideal synchronization of the data is assumed in this paper, so that the differential current is computed thanks to 2 values measured at the same absolute time. The delay corresponding to data propagation through the optical fiber is taken into account.

The fault propagates both ways along the cable, resulting in a perturbation in the current at both ends of the faulty cable, which does not happen necessarily at the same time if the fault is not at the middle of the cable. The differential current is positive as soon as the fault perturbation reaches one end of the faulty cable. For a healthy link, the fault perturbation reaches first one end, leading to a negative front in the differential current, and then the fault reaches the other end. Thanks to a high enough frequency sampling rate, the negative front in the differential current of a healthy link can be detected, and thus the protection blocked. The differential current protection mentioned in the paper takes its decision on the sign of the first front.

\section{BASIC CONCEPT OF THE EMTR THEORY}

In order to examine the applicability of the EMTR to the problem of fault location in transmission lines, let us consider the case of a lossless, two-conductor line (or a single wire above a perfectly conducting ground). As well-known, the Telegrapher's equations describing the wave propagation along the line are given by

$$
\begin{aligned}
& \frac{\partial v(x, t)}{\partial x}+L^{\prime} \frac{\partial i(x, t)}{\partial t}=0 \\
& \frac{\partial i(x, t)}{\partial x}+C^{\prime} \frac{\partial v(x, t)}{\partial t}=0
\end{aligned}
$$

in which $v(x, t)$ and $i(x, t)$ are voltage and current waves along the line, and $L^{\prime}$ and $C^{\prime}$ are the per-unit-length inductance and capacitance of the line, respectively.

Applying the time reversal transformation $t \rightarrow-t$ to the Telegrapher's equations and changing the sign of the current $i(x, t) \rightarrow-i(x,-t)[19]$ yields

$$
\begin{aligned}
& \frac{\partial v(x,-t)}{\partial x}+L^{\prime} \frac{\partial(-i(x,-t))}{\partial(-t)}=0 \\
& \frac{\partial(-i(x,-t))}{\partial x}+C^{\prime} \frac{\partial v(x,-t)}{\partial(-t)}=0
\end{aligned}
$$

It can readily be seen that equations (4)-(5) are formally identical to equations (1)-(2). Therefore, the Telegrapher's equations for lossless lines are invariant under time reversal transformation $[16,19]$.

It is worth noting that, EMTR has been shown to be effective even for lossy lines and in particular for power network transmission lines, which are characterized by a small value for the longitudinal resistance [16].

In general, time reversal process requires that the signals $s_{i}(x, t)$ be measured at multiple observation points during a finite period of time (the so-called time reversal window $\Delta T$ ). However, for the particular case of waves confined in a closed reflecting medium, the time reversal technique has been shown to be efficient with only one observation point [20]. This feature fits the conditions associated with travelling waves in electrical power systems composed of transmission lines terminated by generic power components (i.e., transformers, power converters, etc.) [16].

Moreover, in [16,17], the time reversal window has been chosen long enough so that the recorded transient signal $s(x, t)$ is damped out. In order to relax this assumption, this paper examines the applicability of the EMTR technique with the limited time reversal window for the fault location problem in MTDC networks.

\section{EMTR-BASED FAULT LOCATION ALGORITHM WITH LIMITED TIME REVERSAL WINDOW}

An EMTR-based fault location technique has been successfully applied for locating faults in inhomogeneous networks, radial distribution networks [16] and seriescompensated transmission lines [17]. In these cases, it has been assumed that the fault location system operates after the protection relays maneuver and the time delay of the protection system and the breakers opening time is long enough so that the recorded transient signals are damped out. However, for the case of MTDC networks this assumption is not valid since, as discussed before, the protection system must disconnect the faulted line in less than $10 \mathrm{~ms}$ in order to prevent damage to the power converters [21]. As a consequence, the observation points located at the converter sides are not able to record the full time window of the fault-originated traveling waves.

After the opening of faulted line breakers, the system configuration and the boundary conditions associated with the travelling waves are changed. In this respect, it is worth noting that one of the main hypotheses in time reversal theory is that the system configuration should remain unchanged during the time-reversal process. In addition, the breakers opening introduces additional surges that propagate in the system with additional travelling waves superimposed to the faultoriginated ones. Therefore, the proposed EMTR-based fault location technique has to be suitably modified in order to overcome this problem.

One straightforward approach to solve this problem is to consider a time window of the recorded transient signals prior to the opening of the breakers, so that only the fault originated travelling waves are considered.

As mentioned in Section III, Fink and co-workers [20,22] have shown that, in a closed reflecting medium, it is still possible to locate the source by using a limited time reversal window. In this paper we will take advantage of this feature of the time reversal process for the case of MTDC networks where the fault-originated transient signals are cut due to the protection system and corresponding breaker operations.

The flow-chart of the proposed method is shown in Fig. 2 and illustrates the step-by-step fault location procedure based on EMTR. 


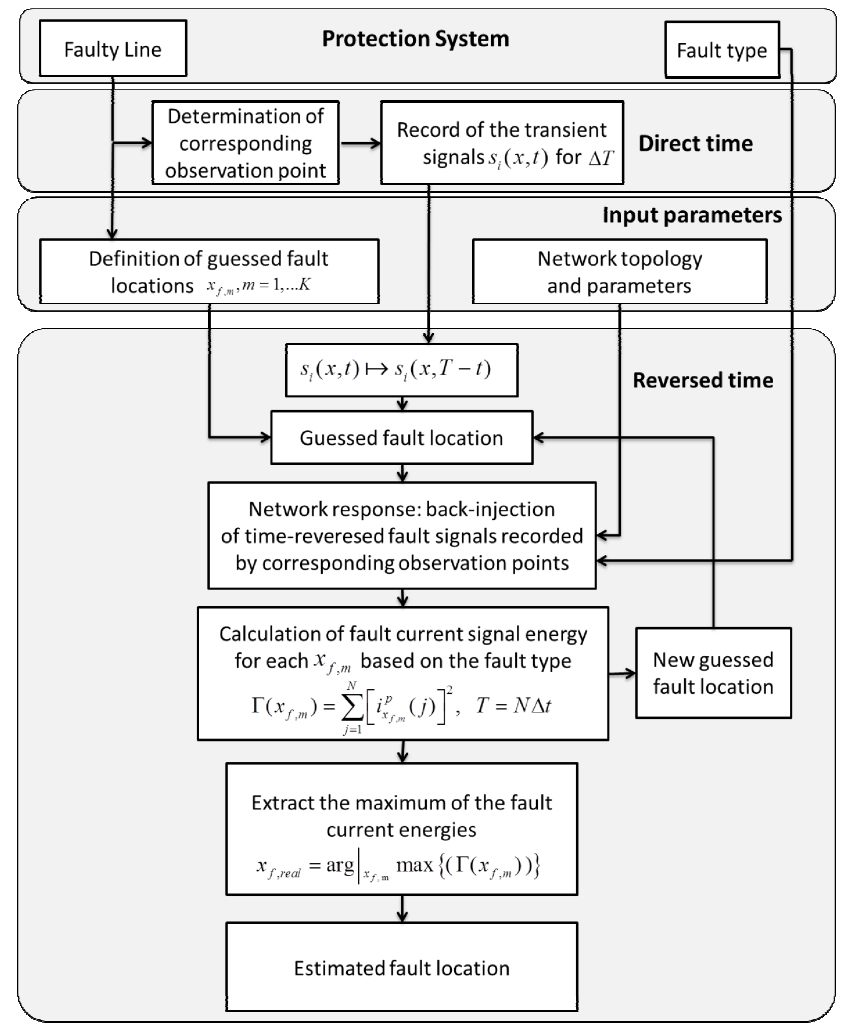

Fig. 2. Flow-chart of the proposed fault location method.

As it can be seen, the proposed procedure requires the knowledge of the network topology as well as its parameters. Such knowledge is used to build a corresponding network model.

It has been assumed that the fault location system operates after the protection system operation described in Section II. Therefore, the faulted line and the fault type have been determined in advance. In particular, we assume that the faulted line detection allows to choose the corresponding observation point to time-reverse the measured transients. This observation point is located at one of the corresponding faulted line ends, which is terminated on power electronics converters.

After the determination of the observation point, fault transients, $s_{i}(t)$ (with $i=1,2$ for positive and negative poles respectively) are recorded in the direct time. It is worth mentioning that we assume these signals are recorded in a finite time window, namely:

$$
s_{i}(t), \quad t \in\left[t_{f}, t_{f}+\Delta T\right]
$$

where $t_{f}$ is the fault inception time, and $\Delta T$ is the time reversal window (in our case it is upper-bounded to $5 \mathrm{~ms}$ ). It is worth observing that this time window is long enough to observe travelling wave reflections coming from all boundaries of the system.

The unknowns of the problem are: (i) the fault type, (ii) its location, and (iii) its impedance. As already mentioned, the fault location system operates after the relaying maneuver and, therefore, the fault type (i.e., pole-to-ground or pole-to-pole) and faulted line are determined in advance. However, as it will be shown in the next section, the proposed method is also able to determine the faulted line without any prior information.

Concerning the fault location, as shown in Fig. 2, we assume a set of $a$ priori locations $x_{f, m}, m=1, \ldots, K$ (corresponding to the faulted line and the rest of the lines) for which the EMTR procedure is applied.

Concerning the fault impedance, as it has been discussed in $[16,17]$, it does not affect the fault location accuracy. Thus, a fixed a- priori fault resistance value has been considered.

In the reversed-time state, the recorded signals are reversed in time and back-injected from the observation point into the system for each guessed location $x_{f, m}$.

It is worth noting that, in order to make the argument of the time-reversed variables positive for the duration of the signal, we have added, in addition to the time reversal, a time delay equal to the duration of the recording time $\Delta T$ :

$$
\begin{aligned}
& \hat{t}=\left(\Delta T+t_{f}\right)-t \\
& \bar{s}(\hat{t}), \hat{t} \in[0, \Delta T]
\end{aligned}
$$

For each of the guessed fault locations and type, we can compute the energy of the signal that corresponds to the currents flowing through the guessed fault location $a^{2}$ :

$$
\Gamma\left(x_{f, m}\right)=\sum_{j=1}^{N}\left[i_{x_{f, m}^{p}}^{p}(j)\right]^{2}, \quad T=N \Delta t
$$

where $N$ is the number of samples, $\Delta t$ the sampling time, and $i_{x_{f, m}}^{p}(j)$ indicates the guessed fault current. The guessed fault current is for each guessed location of three fault types: positive pole-to-ground, negative pole-to-ground and pole-topole.

As mentioned earlier, although the algorithm operates assuming the faulted line known, equation (9) can be calculated for all the lines composing the system. Therefore, the proposed fault location scheme can be used, in principle, as a backup protection to identify the faulted line.

According to the EMTR method, the energy given by (9) is maximized at the position of the fault [16]. Thus, the maximum of the calculated signal energies (of guessed fault current Action Integrals) will indicate the real fault location:

$$
x_{f, \text { real }}=\left.\arg \right|_{x_{f, \mathrm{~m}}} \max \left\{\left(\Gamma\left(x_{f, m}\right)\right)\right\}
$$

It is worth noting that, in [16], the method has been validated by means of an experimental setup that, by nature, includes the presence of measurement noise. Additionally, as shown in [16], the EMTR-based fault location technique was

\footnotetext{
2 it is worth noting that in this case the energy of this current corresponds also to its Action Integral
} 
able to successfully identify the correct fault location even in presence of the measurement noise.

\section{APPLICATION EXAMPLE}

To examine the performance of the proposed method, an application example is considered by making reference to the MTDC network shown in Fig. 1 and adapted from [18].

The network has been simulated in the EMTP-RV simulation environment [23-25] where the lines are represented by constant parameters models (CP).

The lines are assumed to be terminated at on power converters which, for signals characterized by high-frequency spectrum content, can be replaced by high impedances $(100 \mathrm{k} \Omega$ in this study). Such an approximation is justified by the fact that the typical input impedance of HVDC converters on the $\mathrm{DC}$ side is, for frequencies above a few hundreds of $\mathrm{kHz}$, in the order of a few hundreds of $\mathrm{k} \Omega$.

It is worth observing that a detailed model of the converters is not needed since the analyzed time windows are extremely short (i.e., few milliseconds). In this respect, the post fault blocking action of the converter controllers will take place, typically, after the analyzed fault location window.

In Fig. 1, OP1, OP2, OP3, OP4 and OP5 are the observation points corresponding to each node of the network where voltage transients are recorded for both positive and negative poles. Note that the time reversal of the voltage versus current waveforms is equivalent.

As mentioned earlier, the observation point is chosen after the determination of the faulted line by the protection system. In other words, there are two observation points in correspondence of each line where one of them is chosen arbitrary for the fault location problem.

To examine the performance of the proposed method, three fault cases are considered: (i) a pole-to-pole fault at $20 \mathrm{~km}$ of line 1 for a solid fault, (ii) a pole-to-ground fault at $20 \mathrm{~km}$ of line 2 with fault impedance of $10 \Omega$, and (iii) a pole-to-ground fault at $160 \mathrm{~km}$ of line 5 with the fault impedance of $10 \Omega$. In this paper, for the sake of space, the high impedance faults have not been considered. However, as shown in [16], the method is still robust even for the case of high impedance faults.

For each of these cases, the protection system sends the trip signal to the corresponding breakers to disconnect the faulted line. Moreover, a fault occurrence generates travelling waves which propagate along all transmission lines. These transient signals are recorded at the observation point. For the considered fault cases, the observation points are OP1, OP1, and $\mathrm{OP} 5$, respectively.

Fig. 3 shows the recorded pole-to-ground voltage transients for the positive and negative poles in OP1 concerning the fault case \#1. The green line indicates the breaker opening time. As it can be seen, the opening of the breakers changes the behaviour of the travelling waves propagations. The first $5 \mathrm{~ms}$ of these signals, as shown in the zoomed part, are used for the proposed fault location method.
After time reversing the first $5 \mathrm{~ms}$ of the recorded signals, and by assuming the knowledge of the fault type, the same fault type is realized in the time-reversed domain and the position of the guessed fault location is moved along the faulted line as well as for the rest of other lines. Concerning the fault impedance, an a priori fixed value $\left(\mathrm{Z}_{\mathrm{f}}=0 \Omega\right)$ has been assumed.

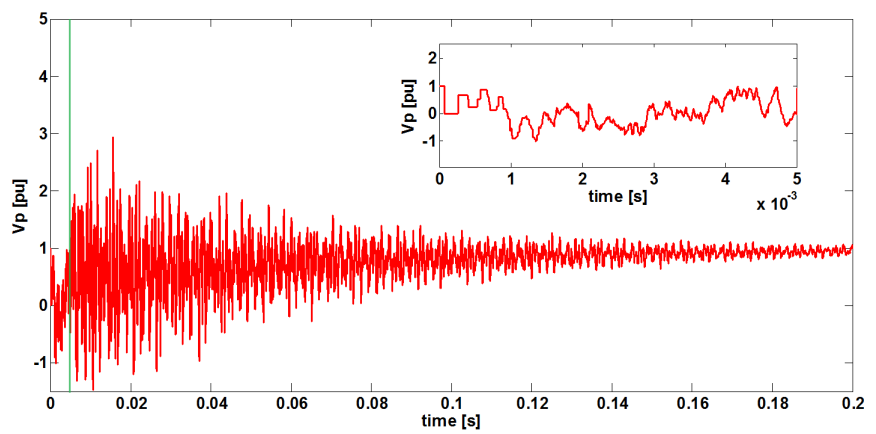

(a)

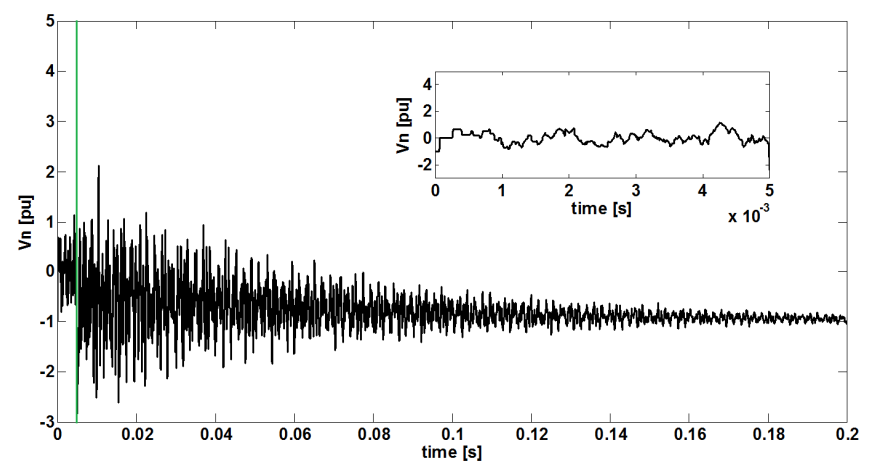

(b)

Fig.3. Recorded transient signals in OP1 for positive (a) and negative (b) poles. The green line shows the breaker opening time amd the inset of figures show the expanded view of the signals for a full-scale of $5 \mathrm{~ms}$.

Figs 4, 5, and 6 show the energy of the fault current signals flowing through the guessed fault points for the considered three fault cases. In these figures, the fault current signal energies are illustrated for the faulted line (solid curve) and the rest of the lines (dashed curves) where, the energy values are normalized to the corresponding peak value of the real fault point.

As it can be observed, the proposed method is remarkably effective in identifying the fault location for all the three fault cases by using limited time reversal window and just a single observation point. In particular, for the considered three fault cases, the fault location error are $1 \mathrm{~km}(1 \%), 200 \mathrm{~m}(0.26 \%)$, and $0 \mathrm{~km}(0 \%)$, respectively.

Further, the proposed method allows the identification of the faulted line correctly. Therefore, it can be used, in principle, as a backup protection system provided that it is deployed into fast-enough computational hardware composed, for instance, by an FPGA-based simulation environment (e.g., [26]). 


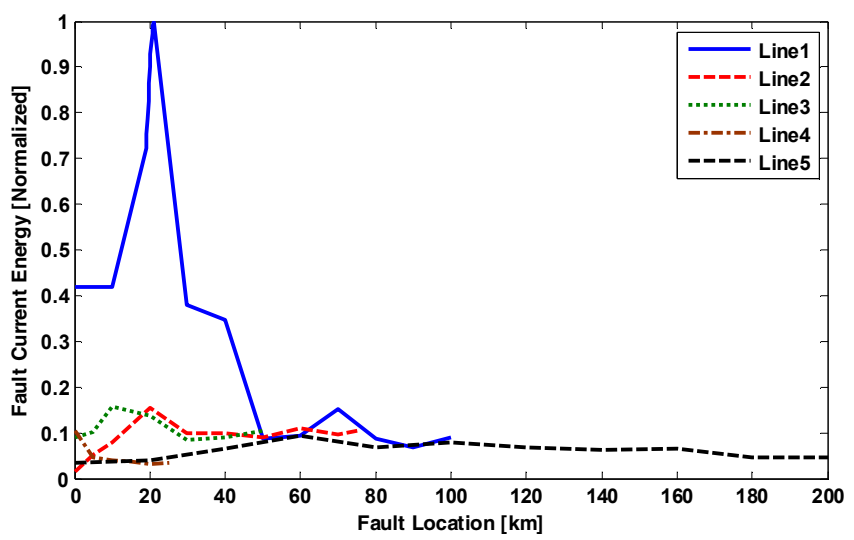

Fig.4. Normalized energy of the fault current signal as a function of the guessed fault location. The real fault is a pole -to-pole solid fault at $x_{\mathrm{f}}=20 \mathrm{~km}$ of line 1 .

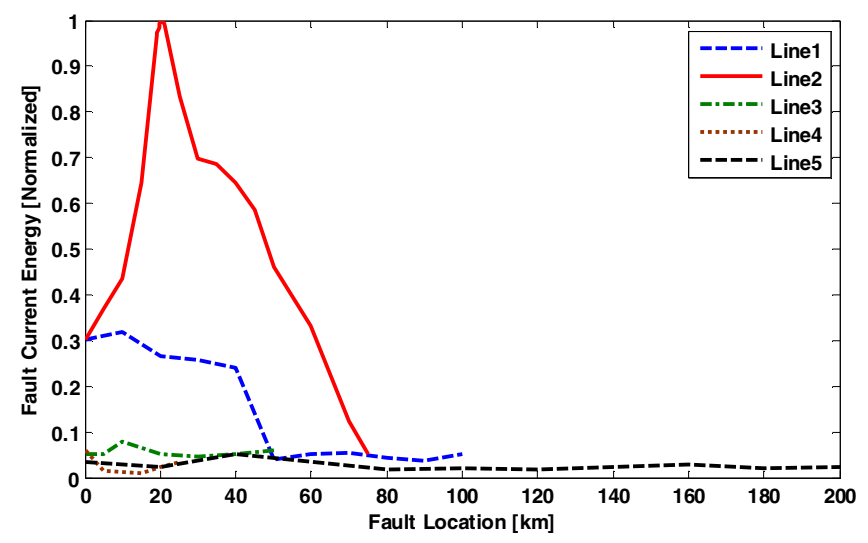

Fig.5. Normalized energy of the fault current signal as a function of the guessed fault location. The real fault is a pole -to-ground fault at $\mathrm{xf}=20 \mathrm{~km}$ of line 2 and the fault impedance is $10 \Omega$.

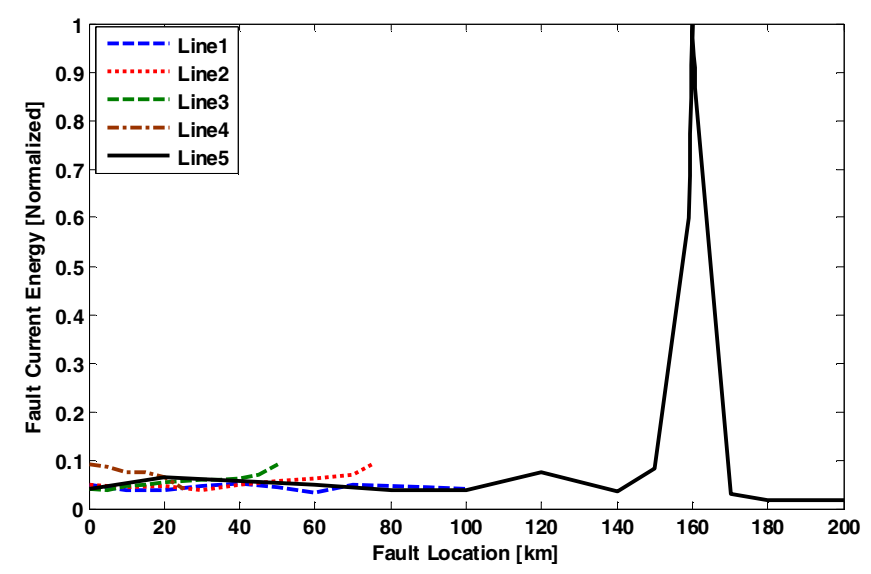

Fig.6. Normalized energy of the fault current signal as a function of the guessed fault location. The real fault is a pole -to-ground fault at $\mathrm{xf}=160 \mathrm{~km}$ of line 5 and the fault impedance is $10 \Omega$.

\section{SENSITIVTY OF THE FAULT LOCATION AS A FUNCTION OF THE TIME-REVERSAL WINDOW LENGTH}

In order to evaluate the effect of time reversal window duration on the accuracy of the EMTR fault location method, an additional sensitivity analysis has been carried out. To this end, for the fault case \#2, the proposed method is applied for five different time reversal window durations, i.e., 3, 4, 5, 7, and $180 \mathrm{~ms}$. The last one corresponds to the time window for which the entire transient is damped out.

The performance of the proposed fault location method for the different time reversal window durations is shown in Fig. 6. It can be observed that the increase in the time reversal window duration results in a narrower peak around the localized fault point, and provides better accuracy. However, windows lengths compatible with HVDC protection needs are still adequate to perform the fault location functionality.

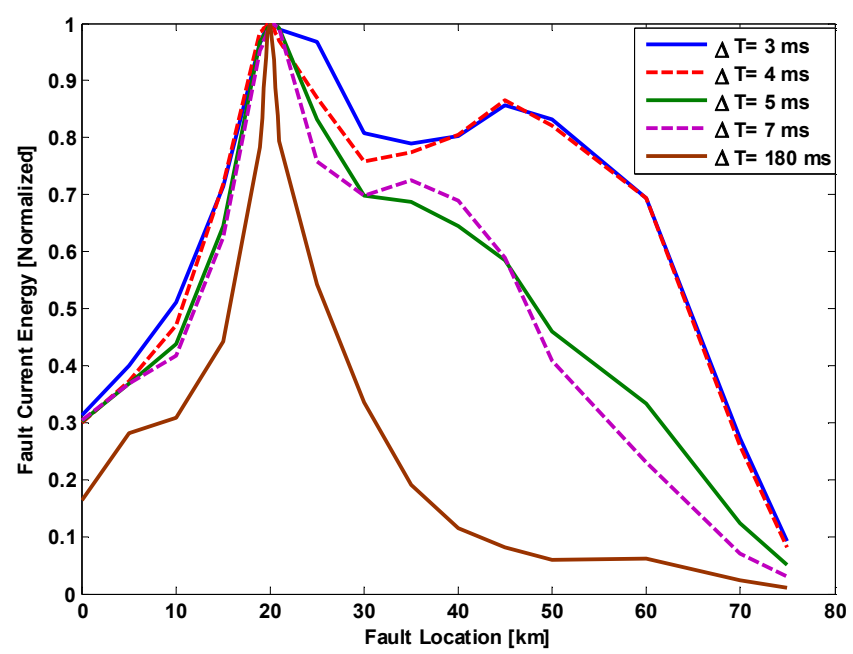

Fig.7. Normalized energy of the fault current signal as a function of the guessed fault location (fault case \#2) for different time-reversal window lengths.

\section{CONCLUSION}

In this paper, the applicability of the fault location method based on the electromagnetic time-reversal theory was presented to the case of multi-terminal DC (MTDC) networks. The EMTR fault location method is integrated in cascade with the MTDC protection scheme recently proposed within the TWENTIES EU project.

The paper has then proposed an MTDC networks fault location scheme composed by the following steps: (1) determination of the observation point based on the corresponding faulted line, (2) measurement of the faultoriginated electromagnetic transient in the single observation point, (3) simulation of the back-injection of the time-reversed measured fault signal for different guessed fault locations using a network model capable of representing traveling-waves, and (4) determination of the fault location by finding, in the network model, the point characterized by the highest energy 
concentration associated with the back-injected time-reversed fault current.

It is worth observing that, by construction, the proposed EMTR fault location scheme requires the use of a single observation point. This peculiarity may represent a major advantage avoiding necessary time synchronization between the MTDC-fault recording stations and the transfer of large amount of fault-transients among the MTDC network faultrecorders.

Additionally, in order to be compatible with the fast action of the MTDC network protections, the paper has discussed the robustness of the EMTR-based fault location scheme with respect to limited time windows used to time reverse the faultoriginated electromagnetic transients. Such an analysis is of particular importance since only few milliseconds of faultoriginated transients can be used before the intervention of the line breakers. In this respect, the sensitivity of the proposed fault location method has been analyzed against such a time reversal window length and its robustness has been proved.

In summary, the proposed fault location scheme exhibits characteristics that justify its applicability also to the case of MTDC networks for which it may represent, also, a valid backup protection scheme provided it is deployed in adequate hardware capable of performing the time-reversal operation in reduced time.

\section{REFERENCES}

[1] J. Suonan, S. Gao, G. Song, Z. Jiao, and X. Kang, "A novel faultlocation method for HVDC transmission lines," IEEE Trans. on Power Del., vol. 25, no. 2, April, 2010.

[2] V. F. Lescale, A. Kumar, L-E. Juhlin, H. Björklund, K. Nyberg, "Challenges with multi-terminal UHVDC transmissions," POWERCON and IEEE Power India Conf., New Delhi, India, 2008.

[3] K. D. Kerf, K. Srivastava, M. Reza, D. Bekaert, S. Cole, D. Van Hertem, R. Belmans, "Wavelet-based protection strategy for DC faults in multiterminal VSC HVDC systems," IET Generation, Transmission \& Distribution., vol. 5, no. 4, pp. 496-503, Sep. 2010

[4] IEEE Std C37.114, "IEEE Guide for Determining Fault Location on AC Transmission and Distribution Lines," 2004.

[5] O. M. K. K. Nanayakkara, A. D. Rajapakse, R. Wachal, "Travelingwave-based line fault location in star-connected Multiterminal HVDC Systems," IEEE Trans. on Power Del., vol. 27, no. 4, Oct. 2012.

[6] O. M. K. K. Nanayakkara, A. D. Rajapakse, R. Wachal, "Location of DC line faults in conventional HVDC systems with segments of cables and overhead lines using terminal measurements," IEEE Trans. on Power Del., vol.27, no.1, pp.279,288, Jan. 2012.

[7] O. Gomis-Bellmunt, J. Liang, J. Ekanayake, R. King, N. Jenkins, "Topologies of multi-terminal HVDC-VSC transmission for large offshore wind farms," Electric Power Systems Research, vol. 81, no. 2, pp. 271-281, Feb. 2011.

[8] M. Fink, C. Prada, F. Wu and D. Cassereau, "Self focusing with time reversal mirror in inhomogeneous media", IEEE Ultrason. Symp., pp. 681-686, 1989.

[9] M. Fink, "Time reversal of ultrasonic fields. I. basic principles", IEEE Trans. on Ultrasonics, Ferroelectrics and Frequency Control, vol. 39, issue 5, Sept. 1992, pp. $555-566$.
[10] F. Wu, J.-L. Thomas, M. Fink, "Time reversal of ultrasonic fields. II: experimental results", IEEE Trans. on Ultrasonics, Ferroelectrics and Frequency Control, vol. 39, issue 5, Sept. 1992, pp: $567-578$.

[11] D. Cassereau, M. Fink, "Time-reversal of ultrasonic fields. III. theory of the closed time-reversal cavity", IEEE Trans. on Ultrasonics, Ferroelectrics and Frequency Control, vol. 39, issue 5, Sept. 1992, pp: $579-592$.

[12] G. Lerosey, J. de Rosny, A. Tourin, A. Derode, G. Montaldo, M. Fink, "Time reversal of electromagnetic waves", Phys. Rev. Lett. 92, pp. 193904-193901-193903, 2004.

[13] D. Liu; G. Kang; L. Li; Y. Chen; S. Vasudevan, W. Joines, Q. Huo Liu, J. Krolik, L. Carin, "Electromagnetic time-reversal imaging of a target in a cluttered environment", IEEE Trans. Antennas Propagat., vol. 53, pp. 3058-3066, 2005.

[14] H. Zhai, S. Sha, V. K. Shenoy, S. Jung, M. Lu, K. Min, S. Lee, D.S. Ha, "An electronic circuit system for time-reversal of ultra-wideband short impulses based on frequency-domain approach", IEEE Transactions on Microwave Theory and Techniques, , vol. 58, issue: 1, pp. 74-86, 2010.

[15] N. Mora, F. Rachidi, M. Rubinstein, "Application of the time reversal of electromagnetic fields to locate lightning discharges," Journal of Atmospheric Research, Vol. 117, pp. 78-85, 2012.

[16] R. Razzaghi, G. Lugrin, H. M. Manesh, C. Romero, M. Paolone, and F. Rachidi, "An efficient method based on the electromagnetic time reversal to locate faults in power networks," IEEE Trans. on Power Del., vol. 28, pp. 1663-1673, 2013.

[17] R. Razzaghi, G. Lugrin, M. Paolone, F. Rachidi, "On the use of electromagnetic time reversal to locate faults in series-compensated transmission lines," Proceedings of the 2013 IEEE Grenoble PowerTech, IEEE Power Engineering Society and Grenoble Institute of Technology, pp. 1-5, June, 2013.

[18] J. Descloux, B. Raison, J. B. Curis, “ Protection strategy for undersea MTDC grids," Proceedings of the 2013 IEEE Grenoble PowerTech, IEEE Power Engineering Society and Grenoble Institute of Technology, pp. 1-6, June, 2013.

[19] F. Rachidi, M. Rubinsein, "Time reversal of electromagnetic fields and its application to lightning location" 12th International Symposium on Lightning Protection SIPDA, Belo Horizonte, Brazil, October. 7-11, 2013.

[20] C. Draeger, J-C. Aime, M. Fink, "One-channel time-reversal in chaotic cavities: experimental results," Journal of the Acoustical Society of America - J ACOUST SOC AMER, 105(2), pp. 618-625, 1999.

[21] J. Descloux, C. Gandioli, B. Raison, N. Hadjsaid, P. Tixador, "Protection system for meshed HVDC network using superconducting fault current limiters," Proceedings of the 2013 IEEE Grenoble PowerTech, IEEE Power Engineering Society and Grenoble Institute of Technology, pp. 1-5, June, 2013.

[22] M. Fink, "Time-reversal acoustics in complex environments," geophysics, Vol. 71, No. 4,pp. I151-I164, 2006.

[23] H. W. Dommel: "Digital computer solution of electromagnetic transients in single and multiphase networks", IEEE Transactions, Vol. PAS-88, pages 388-399, April 1969.

[24] J. Mahseredjian, S. Lefebvre and X.-D. Do, "A new method for timedomain modelling of nonlinear circuits in large linear networks", Proc. of 11th Power Systems Computation Conference PSCC, August 1993.

[25] J. Mahseredjian, S. Dennetière, L. Dubé, B. Khodabakhchian and L. Gérin-Lajoie: "On a new approach for the simulation of transients in power systems". Electric Power Systems Research, Volume 77, Issue 11, September 2007, pp. 1514-1520.

[26] R. Razzaghi, M. Paolone, F. Rachidi, “A general purpose FPGA-based real-time simulator for power systems Applications," Proceedings of IEEE $4^{\text {th }}$ European Innovative Smart Grid Technologies (ISGT), Copenhagen, Denmark, October, 2013. 